Supercritical Fluid Conditions," p. 101, Ann Arbor Science Publishers, Michigan (1983).

5) Marina, J. M. and D. P. Tassios: Ind. Eng. Chem. Proc. Des. Develop., 12, 271 (1973).

6) McHugh, M. A., M. W. Mallet and J. P. Kohn: "Chemical Engineering at Supercritical Fluid Conditions," p. 113, Ann Arbor Science Publishers, Michigan (1983).

7) Nakayama, T., H. Sagara, K. Arai and S. Saito: Fluid Phase Equilibria, 38, 109 (1987).

8) Othmer, D. F., W. S. Bergen, N. Shlechter and P. F. Bruins: Ind. Eng. Chem., 37, 9, 890 (1945).

9) Paulaitis, M. E., R. G. Kander and J. R. DiAndreth: Ber. Bunsenges. Phys. Chem., 88, 869 (1984).

10) Renon, H. and J. M. Prausnitz: AIChE J., 14, 135 (1968).

11) Sagara, H.: Preprint of the Fukuoka meeting of the Society of
Chemical Engineers, Japan, S-C6 (1983)

12) S $\phi$ rensen, J. M. and W. Arlt: "Liquid-Liquid Equilibrium Data Collection," p. 236, DECHEMA (1980).

13) Takishima, S., K. Saiki, K. Arai and S. Saito: J. Chem. Eng Japan, 19, 48 (1986).

14) Treybal, R. E., L. D. Weber and J. F. Daley: Ind. Eng. Chem., 38, 817 (1946).

15) Treybal, R. E.: "Liquid Extraction," p. 30, McGraw-Hill Book Company, Inc. (1963).

(A part of this work was presented at the 19th Autumn Meeting of the Society of Chemical Engineers, Japan, at Nagoya, 1985 and at The 51st Annual Meeting of The Society of Chemical Engineers, Japan, at Osaka, 1986.)

\title{
LASER-DOPPLER MEASUREMENTS OF TURBULENCE STRUCTURE IN A DRAG-REDUCING PIPE FLOW WITH POLYMER INJECTION
}

\author{
HIROMOTO USUI, MATSURU KODAMA AND YUJI SANO \\ Department of Chemical Engineering, Yamaguchi University, Ube 755
}

Key Words: Drag Reduction, Polymer Injection, Turbulence, Laser Doppler Velocimeter, Polymer Additive

\begin{abstract}
Aqueous solutions of polyethylene oxide were injected into a pipe flow through a small tube at the center of a pipe. The turbulent characteristics in drag-reducing flow with polymer injection were measured by means of a Laser Doppler Velocimeter (LDV). The experimental results were compared with measurements both in a Newtonian fluid (water) flow and in a premixed flow with $300 \mathrm{ppm}$ homogeneous polymer solution.

The experimental results suggested that the polymer injection caused a thickening of the buffer layer, enlargement of macroscale turbulent eddy and suppression of fine turbulent eddy. A difference in turbulent characteristics between premixed and polymer injected systems was observed in the distributions of turbulent macroscale, skewness factor and flatness factor. The enlargement of macroscale was more significant in the turbulent core region in the case of polymer injection experiments. The measurements of skewness and flatness factors showed that the low-speed fluid element caused by the ejection process passed more clearly the measuring position near the outer boundary of the buffer layer in the case of polymer injection experiments.
\end{abstract}

\section{Introduction}

Drag reduction by use of a polymer additive is important as a power-saving technology in fluid transportation. In 1979, the Trans-Alaska Pipe-Line introduced the use of polymer injection technology to reduce pumping power consumption. Other applications such as flow improvement in sewer systems and slurry transportation have also been reported recently. Dosing of polymer additives into the tube flow is usually accomplished by injecting a relatively concentrated polymer solution by means of a suitable

Received July 3, 1987. Correspondence concerning this article should be addressed to H. Usui. M. Kodama is now with Diafoil Co., Lid. dosing system. Injected polymer solution does not mix soon with the main flow. Heterogeneous flow condition is maintained over a considerably long downstream distance from the polymer dosing station.

Drag reduction in premixed, i.e. homogeneous, dilute polymer solutions has been well documented by many investigations published during the last two decades. However, reports on heterogeneous drag reduction through polymer injection are rather scarce. McComb and Rabie ${ }^{10}$ made precise LDV measurements in a heterogeneous drag-reducing flow with centerline polymer injection. Their experimental results showed that the change of turbulent flow charac- 
teristics was almost the same as those observed in premixed drag-reducing flow. ${ }^{111.14)}$ They observed a thickening of the elastic sublayer, enlargement of the integral scale of auto-correlation, decrease of highfrequency energy spectra and increase of bursting time. Other LDV measurements ${ }^{2-4)}$ partially confirmed the above-mentioned observations obtained by McComb and Rabie. ${ }^{10)}$ However, precise comparison of previous experimental results ${ }^{2-4,10}$ ) has revealed that there existed an essential difference in the interpretation of polymer-turbulent eddy interaction between the previous investigations. McComb and Rabie $^{9)}$ found that the increase in local drag reduction caused by polymer injection corresponded well to the rate of radial spreading of injected polymer into the near-wall region. LDV measurements by McComb and Rabie ${ }^{10}$ showed essentially the same turbulent characteristics as those obtained for premixed system. They concluded that the polymer molecule interacted with the turbulence in an annulus which was near but not at the wall. This is the same reasoning for drag reduction as was proposed for premixed system by previous investigators, ${ }^{1,2,11,17)}$ although the detailed mechanism of polymer-turbulent eddy interaction may be different in the two systems. Bewersdorff ${ }^{3)}$ showed that the velocity profile in the turbulent core of polymer injected flow was not parallel to the Newtonian line when the velocity profile was plotted as $U^{+}$vs. $y^{+}$. On the other hand, the velocity profile in premixed system showed a parallel shift to the Newtonian line. Bewersdorff suggested that the turbulent structure of polymer injected flow was changed even in the turbulent core region. This observation was confirmed by Berman and Sinha. ${ }^{21}$ They also showed that the distribution of skewness and flatness factors were a little different from the Newtonian and premixed lines. Berman and Sinha concluded that the fluctuation of large eddy in the turbulent core of polymer injected flow pushed more fast-moving fluid to the wall.

Although experimental results for drag reduction through polymer injection have been accumulated as mentioned above, the difference between heterogeneous polymer injection system and premixed dragreducing system is still obscure. More precise observation of the interaction between the turbulent eddy and polymer thread in heterogeneous drag-reducing systems is needed to establish a design guideline for the application of polymer-injection technology. The purpose of this study is to obtain a set of turbulence measurement results by Laser Doppler velocimetry in a drag-reducing pipe flow with polymer injection. The influence of injected polymer threads on the turbulence structure will be discussed.

\section{Experimental Apparatus and Procedure}

The experimental setup was the same as reported in the previous work ${ }^{16)}$ except for the test tube. A oncethrough flow system including a large storage tank $\left(1.5 \mathrm{~m}^{3}\right)$ was employed to minimize the mechanical degradation of polymer additives. The test section was an $8 \mathrm{~m}$ long $\times 51.3 \mathrm{~mm}$ i.d. horizontal PVC tube which formed part of a once-through flow system. For the polymer injection experiments, water was forced to flow through the head tank, and a relatively concentrated polymer solution contained in a pressurized vessel was injected through an injection nozzle. The center-line injection mode was accomplished by a single nozzle $(4 \mathrm{~mm}$ i.d.). The inner diameter of injection nozzle was designed so that the outlet velocity from the injection nozzle $\left(U_{p}=\right.$ $0.267 \mathrm{~m} / \mathrm{s}$ ) coincided approximately with the centerline velocity of the tube $\left(U_{c}=0.242 \mathrm{~m} / \mathrm{s}\right)$ at $R e=10^{4}$. The pressure drop along the test tube was measured by means of $\mathrm{CCl}_{4} U$-tube manometers at $x / D=$ 30-300.

The LDV (laser Doppler velocimeter) was used in the forward-scattering, reference beam mode. The LDV measuring section located $7 \mathrm{~m}(x / D=136)$ downstream from the injection point was a transparent polyester film ( $40 \mu \mathrm{m}$ thickness) which was set up like the apparatus of Mizushina and Usui. ${ }^{11)} \mathrm{A}$ $5 \mathrm{~mW} \mathrm{He}-\mathrm{Ne}$ laser was used to measure streamwise velocity component. The laser beam diameter, $\sigma$, at the measuring point is, for convenience, estimated by the following equation, given elsewhere (e.g. George and Lumley ${ }^{6)}$ ):

$$
\sigma=\frac{2^{1 / 2}}{\pi}-\frac{f_{0} \lambda}{d_{0}}
$$

In this work the focal length of the lens, $f_{0}$, wave length, $\lambda$, and the diameter of the laser beam at the lens, $d_{0}$, were $250 \mathrm{~mm}, 0.6328 \mu \mathrm{m}$, and $1 \mathrm{~mm}$ respectively. The scattering volume is usually approximated by an ellipsoid of which the major axis length is $\sigma_{1}$ and the lengths of shorter axes are $\sigma_{2}$ and $\sigma_{3}$. Using the above-mentioned values and the value of the cross angle of laser beams at the measuring point $(\theta=5.98$ degrees), $\sigma_{1}, \sigma_{2}$ and $\sigma_{3}$ were estimated as $1.38,0.072$ and $0.072 \mathrm{~mm}$ respectively. The major axis of scattering volume was oriented in the spanwise direction of the test pipe. The signal processor (frequency-tovoltage converter) is essentially the same as that described by Mizushina and Usui. ${ }^{11)}$ The instantaneous longitudinal velocity signal was recorded by a data recorder (TEAC R-61). The voltage outputs of the instrument were digitized and stored on magnetic tape. The sampling interval and the number of data were 0.005 second and 30,000 , respectively. Statistical processing of the digitized data was made with an 
Table 1. Experimental conditions for LDV measurements

\begin{tabular}{lcccccccc}
\hline & $\begin{array}{c}c_{p} \\
{[\mathrm{ppm}]}\end{array}$ & $\begin{array}{c}c_{a v} \\
{[\mathrm{ppm}]}\end{array}$ & $\begin{array}{c}v \\
{\left[\mathrm{~cm}^{2} / \mathrm{s}\right]}\end{array}$ & $\begin{array}{c}U_{a v} \\
{[\mathrm{~cm} / \mathrm{s}]}\end{array}$ & $\begin{array}{c}R e \\
{[-]}\end{array}$ & $\begin{array}{c}f \\
{[-]}\end{array}$ & $\begin{array}{c}u^{*} \\
{[\mathrm{~cm} / \mathrm{s}]}\end{array}$ & $\begin{array}{c}D R \\
{[\%]}\end{array}$ \\
\hline Water & - & - & 0.0108 & 23.5 & 11,200 & 0.00684 & 1.37 & - \\
Injection & 4000 & 30 & 0.0108 & 21.0 & 9,900 & 0.00313 & 0.829 & 60.5 \\
Premix & - & 300 & 0.0163 & 30.9 & 9,700 & 0.00380 & 1.35 & 52.2 \\
\hline
\end{tabular}

ACOS-850 computer system at Yamaguchi University.

Polyethylene oxide (grade Alcox E-160 supplied by Meisei Chemical Corp.) was used as polymer additive. Concentrated polymer solution containing $4000 \mathrm{ppm}$ Alcox E-160 was prepared. It was pressurized for injection through the injection nozzle into the test tube. For premixed drag reduction experiments, a dilute polymer solution $\left(c_{a v}=300 \mathrm{ppm}\right)$ was prepared in a large storage tank $\left(1 \mathrm{~m}^{3}\right)$. This polymer solution was used for once-through drag reduction experiments. A stabilizer, (Sandex-C, $30 \mathrm{ppm}$ ) to prevent chemical degradation of the polymer additives was used in this work. Also, fresh milk (300 ppm) was added as scattering particles for LDV measurements.

\section{Experimental Results and Discussion}

\subsection{Drag reduction}

It was intended to measure the turbulence structure in heterogeneous polymer injected flow, premixed drag-reducing flow and a Newtonian fluid (water) flow at the same Reynolds number. Also, we intended to compare the data of heterogeneous and of premixed systems at the same drag reduction rate. The drag reduction rate is defined as:

$$
D R[\%]=\frac{f_{N}-f_{P}}{f_{N}} \times 100
$$

where $f_{N}$ and $f_{P}$ are the friction factor of Newtonian fluid (water) flow and that of drag-reducing system respectively. We selected the experimental conditions for polymer injection experiments as an injected polymer concentration of $c_{p}=4000 \mathrm{ppm}$ and a bulk averaged polymer concentration of $c_{a v}=30 \mathrm{ppm}$. The results of friction factor measurements are shown in Fig. 1. For a comparison of the drag reduction data of the premixed system at $c_{a v}=30 \mathrm{ppm}$ with those obtained for polymer injection experiments, the drag reduction ratio would be too low, as indicated by solid square symbols in Fig. 1, because of the pipe diameter effect in the premixed system. Therefore, after some trial experiments we selected $c_{a v}=300 \mathrm{ppm}$ as the polymer concentration in the premixed system. The drag reduction data at this concentration show almost the same drag reduction ratio as indicated in Fig. 1. The experimental conditions of LDV measurements are shown in Table 1. For heterogeneous

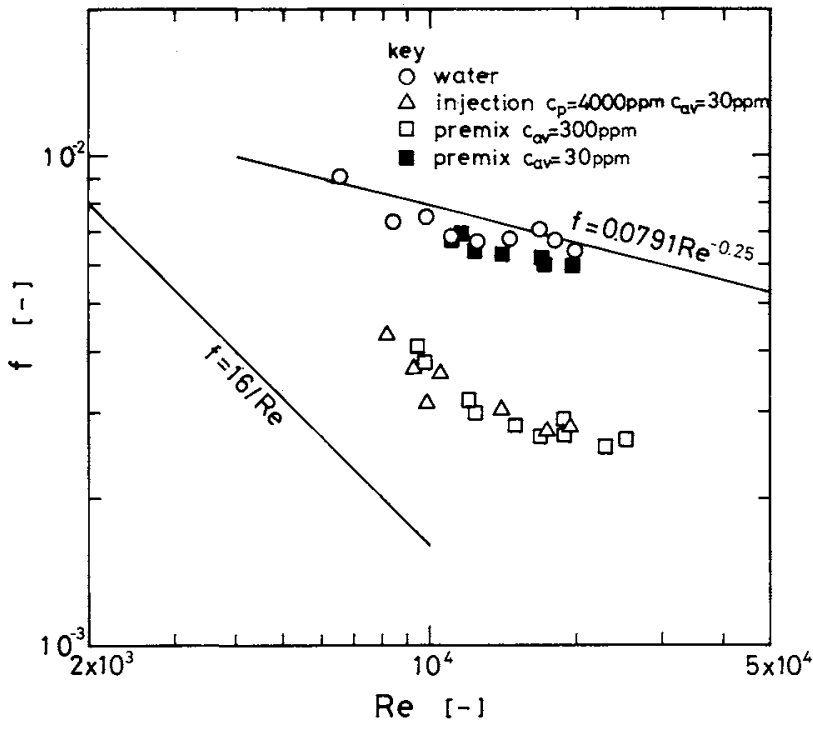

Fig. 1. Friction factor, $f$, as function of Reynolds number, $R e$, using $51.3 \mathrm{~mm}$ i.d. test pipe

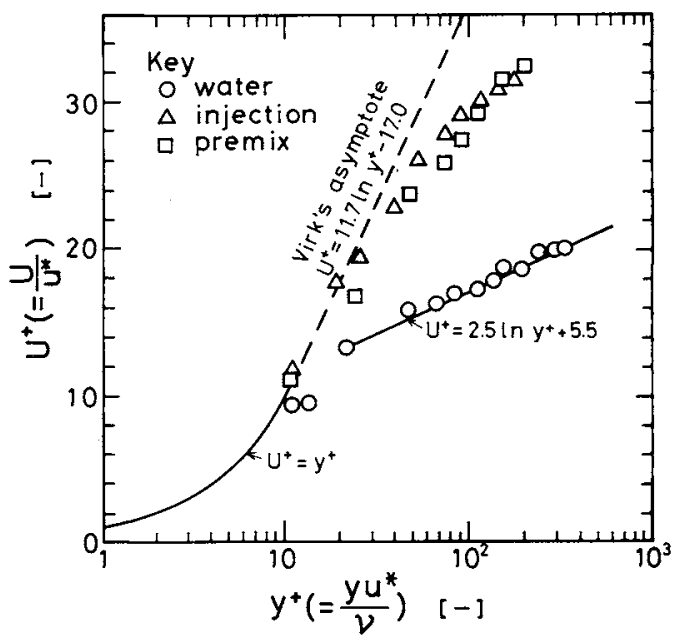

Fig. 2. Mean velocity profiles

polymer injection experiments, the viscosity of water was used to calculate the Reynolds number in heterogeneous system. Because of the viscosity increase in $300 \mathrm{ppm}$ premixed polymer solution, the value of friction velocity, $u^{*}$, was almost the same as that of water although the drag reduction ratio reached $52.5 \%$.

\subsection{Velocity distribution}

The distributions of streamwise velocity, $U$, are shown in Fig. 2. The velocity distributions of both 
polymer injection and premixed systems show the increase in buffer layer thickness. But they show almost the same velocity profiles. Berman ${ }^{2)}$ and Bewersdorff ${ }^{3)}$ have reported that the slope of the velocity profile with polymer injection in turbulent core was different from the slope for a Newtonian fluid when nondimensionalized velocity, $U^{+}$ $\left(=U / u^{*}\right)$, was plotted against $\ln y^{+}$. The present experimental results do not show this point clearly. The velocity profile with polymer injection shown in Fig. 2 is rather similar to the velocity profile obtained by McComb and Rabie. ${ }^{10)}$ We can only say that, if the drag reduction level is almost the same $(D R=$ $50-60 \%$, the velocity profile observed in polymer injection experiments is very similar to that obtained for the premixed drag-reducing system.

\subsection{Turbulent intensity}

The turbulent intensity profiles obtained are shown in Fig. 3. One of the most reliable measurements of turbulent intensity for a Newtonian fluid at lower Reynolds number has been given by Pennel et al., ${ }^{13)}$ and their result is compared with ours in this diagram. The turbulent intensity of a Newtonian fluid (water) of this work is nearly half the previously wellestablished value for all the radial positions of a tube. This discrepancy was interpreted as the result of larger sampling volume of the present LDV system compared with the microscale of turbulence. The correction of measured turbulent intensity based on the spatial resolution analysis by George and Lumley ${ }^{6}$ was made. The detailed correction method was described in the previous paper. ${ }^{16)}$ The corrected data shown by the open-circle symbol in Fig. 3 are in reasonable agreement with the measurements by Pennel et al. ${ }^{13)}$ except near the wall.

For drag-reducing systems, it is well accepted that the flow-stabilizing effect of polymer additive causes enlargement of the turbulence scale at lower frequency and causes the suppression of micro-eddies. This means that the same finite scattering volume of a LDV system can measure more correctly the turbulent intensity in drag-reducing flow. Precise correction for the turbulent intensity in drag-reducing system was not possible, because no measurements of the rate of dissipation were made in this work. The upward shift of turbulent intensity profiles of both polymer injection and premixed systems from the case of a Newtonian fluid (water) observed in Fig. 3 does not mean a true increase of turbulent intensity, even at the same friction velocity. Because the absolute value of turbulent intensity depends strongly on the relationship between the size of scattering volume and the turbulence scale, we can only say, from the results shown in Fig. 3, that the peak location of the turbulent intensity profile in the drag-reducing system is shifted a little to the position at $y^{+}=25-30$.

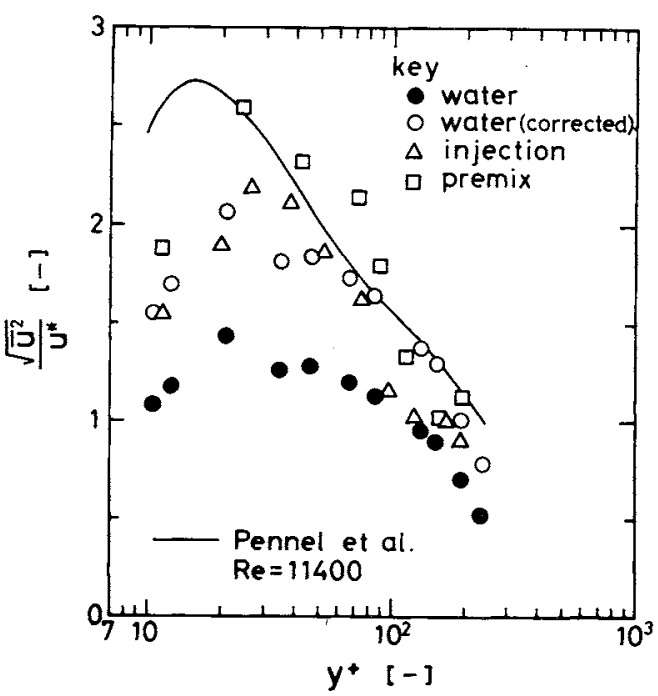

Fig. 3. Turbulent intensity profiles

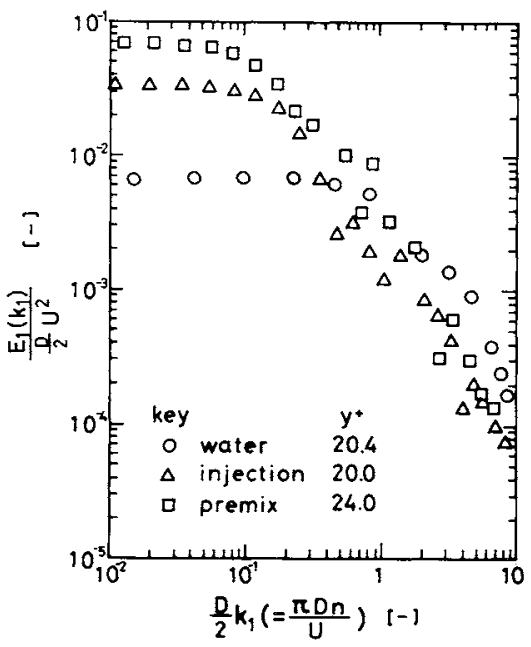

Fig. 4. Distribution of one-dimensional energy spectrum scaled by bulk flow parameters

\subsection{Effect of polymer injection on streamwise energy spectrum}

The experimental results of one-dimensional streamwise energy spectra, $E_{1}\left(k_{1}\right)$, near the wall are shown in Fig. 4. The wave number, $k_{1}$, is defined as $k_{1}=2 \pi n / U$, where $n$ and $U$ are the frequency and local velocity, respectively. It was confirmed that correction of the energy spectrum was not significant if the frequency was less than $5-10 \mathrm{~Hz}$; e.g. see Fig. 6 of ref. 16. This frequency range corresponds to $(D / 2) k_{1}=4$ 8 . We can conclude that, although the high-frequency fluctuating velocity in water flow is not accurate, the energy spectra obtained for drag-reducing flow in this work is accurate enough for study of the effect of polymer additive on turbulence structure. This may be due to the enlargement of turbulent eddies compared with the finite scattering volume of the LDV system.

The energy spectra in drag-reducing systems shown 
in Fig. 4 indicate the upward shift from the water data in the lower frequency range. On the other hand, damping of high-frequency eddies is observed in the case of drag-reducing flows. The above-mentioned observations correspond to the experimental evidence reported by McComb and Rabie ${ }^{(0)}$ and El-Riedy and Latto. ${ }^{4)}$ The difference between polymer injection experiments and premixed drag reduction at higher frequency is not clear because of experimental error. But the clear difference in the upward shift of the energy spectrum in the lower-frequency region is observed in Fig. 4. The level of upward shift is evaluated by the macroscale of turbulence, $A_{f}$, defined by:

$$
\lim _{n \rightarrow 0} \frac{1}{4 \bar{u}^{2}} E_{1}(n)=\frac{1}{U} \int_{0}^{\infty} f(x) d x=\Lambda_{f} / U
$$

where $f(x)$ is the longitudinal space correlation coefficient. The experimental results of macroscale are shown in Fig. 5. The macroscales in a Newtonian (water) flow coincide with Kudva and Sesonske's data. ${ }^{7}$

In the case of premixed drag reduction, the macroscale shows a high degree of enlargement $\left(\Lambda_{f} / D \approx 2.5\right)$. It has been well accepted that premixed polymer additive caused the enlargement both of wall turbulence scale $^{5}$ and bursting time. ${ }^{1,11}$ Fortuna and Hanratty ${ }^{5}$ reported that the ratio of nondimensionalized transverse macroscale, $\Lambda_{z}^{+}$, of premixed dragreducing system to that of a Newtonian fluid flow was equal to 6.7 when drag reduction rate was $64.5 \%$. The experimental results of $A_{f}$ shown in Fig. 5 give the nondimensionalized macroscale ratio as:

$$
\frac{\Lambda_{f, \text { premix }}^{+}}{\Lambda_{f, \text { water }}^{+}}=4
$$

in all the radial positions, where $\Lambda_{f}^{+}$is defined as $\Lambda_{f}^{+}=\Lambda_{f} u^{*} / v$. The ratio given above corresponds to the experimental data of Fortuna and Hanratty ${ }^{5}$ if we take into account the present drag reduction ratio, i.e. $D R=52.2 \%$. In the case of heterogeneous polymer injected drag reduction, the macroscale is enlarged to $\Lambda_{f} / D=1-2$ near the wall. The macroscale in the polymer injected flow is more enlarged in the turbulent core. The experimental evidence that the macroscale of turbulence in the turbulent core is more enlarged in the polymer injection system indirectly supports the possibility that the polymer threads interact with turbulent eddy in the turbulent core region. However, it is not clear why the same level of drag reduction can occur in the polymer injection system in spite of the different amount of enlargement in macroscale if it is compared with the premixed system.

\subsection{Skewness factor and flatness factor}

Details of the fluctuations are summarized in the

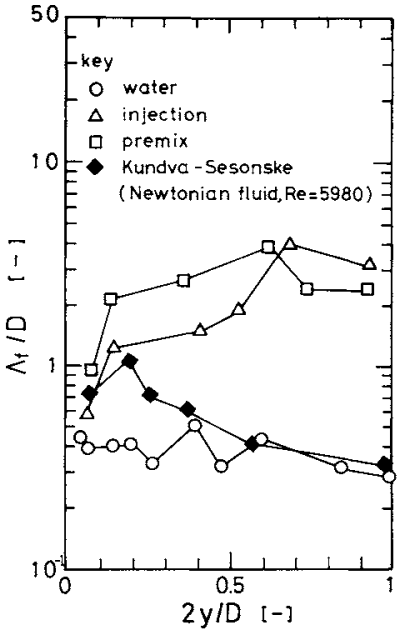

Fig. 5. Distribution of macroscale, $\Lambda_{f}$, of turbulent eddy

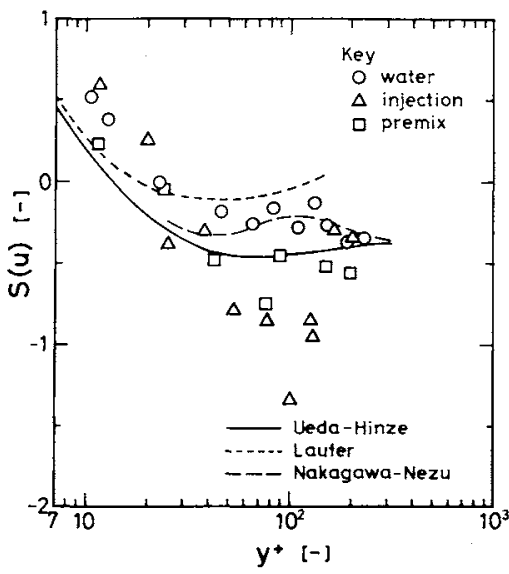

Fig. 6. Distribution of the skewness factor of $u$

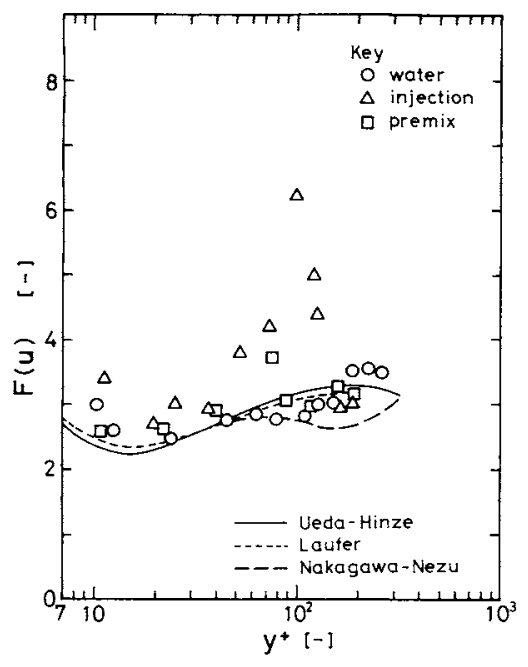

Fig. 7. Distribution of the flatness factor of $u$

distributions of skewness and flatness factors in Figs. 6 and 7. The skewness factor and the flatness factor of streamwise fluctuating velocity are defined as:

$$
\begin{gathered}
S(u)=\bar{u}^{3} /\left(\bar{u}^{2}\right)^{3 / 2} \\
F(u)=\bar{u}^{4} /\left(\bar{u}^{2}\right)^{2}
\end{gathered}
$$


The results for water shown in Figs. 6 and 7 are in reasonable agreement with the data of Laufer ${ }^{8}{ }^{8}$ Ueda and Hinze ${ }^{15)}$ and nakagawa and Nezu. ${ }^{12)}$ The results of drag-reducing flow show almost the same distribution as a Newtonian fluid very near the wall, but the skewness factor decreases anomalously at $y^{+}=$ $50-120$, and the flatness factor shows a remarkable increase at the same position. In particular, it should be noted that the polymer injection causes a more significant deviation of skewness and flatness factor distributions from the Newtonian distribution. Berman and Sinha ${ }^{2)}$ measured the skewness and flatness factors in both premixed and polymer injected drag reduction. They showed that the skewness factor of the premixed drag-reducing system was lower than the Newtonian value at $y^{+}=60-150$. This observation agrees with the present results. The interaction between premixed polymer additive and wall turbulence causes the enlargement of bursting scale. ${ }^{1,11}$ The low-speed fluid element which is ejected from the wall to the center of the tube may become more significant, and this low-speed signal may cause the decrease of the skewness factor. On the other hand, if we compare the results of polymer injection experiments, there is a great discrepancy between this work and that of Berman and Sinha. The experimental results shown in Figs. 6 and 7 indicate that the appearance of a lowspeed fluid element is detected more typically. But Berman and Sinha showed that the positive skewness persisted much further from the wall than the Newtonian case, and it coincided with the Newtonian value in the center of the tube. They suggested that the fluctuation of the polymer thread in the center of the tube pushed more fast-moving fluid to the wall. Berman and Sinha measured the fluctuating velocity at $x / D=108$ in a $12.7 \mathrm{~mm}$ i.d. test tube. Therefore, flow visualization experiments were carried out to examine the difference of polymer thread-turbulence interaction for different pipe diameters. Two tubes, of which the inner diameters were 12.3 and $51.3 \mathrm{~mm}$, were used as test tubes. The flow behavior of injected polymer thread coloured by indian ink was observed photographically. The results are shown in Fig. 8. The single thread injected at the center of the tube is observed at $x / D=8$ for both $D=51.3$ and $D=$ $12.3 \mathrm{~mm}$. At downstream positions in the larger tube (at $x / D=136$ and $D=51.3 \mathrm{~mm}$ ), fine threads of polymer solutions are observed. They are distorted and spread out radially because of the interaction between turbulence eddies. On the other hand, the polymer thread injected into a smaller tube is not distorted significantly. Although the injected polymer solution is subdivided into several threads at $x / D=300$, all polymer threads seem to flow in the furbulent core region of tube flow. It should be emphasized that the drag reductions obtained for different tube diam-

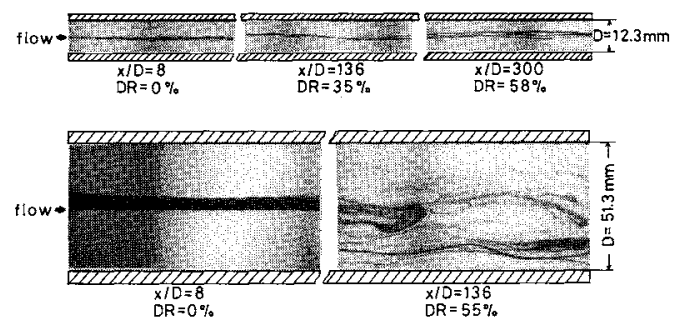

Fig. 8. Flow visualization results of centerline injection of dyed polymer solution at $R e=10^{4}, c_{p}=4000 \mathrm{ppm}$ and $c_{a v}=$ $30 \mathrm{ppm}$

eters are almost the same as shown in this diagram. Taking account of this experimental evidence, we suppose that the injected polymer thread was not subdivided so much in Berman and Sinha's experiments, and the polymer thread flowing in the turbulent core region mainly interacted with turbulent eddy. Also, the measuring position at $x / D=108$ in Berman and Sinha's experiments is not far enough downstream to obtain the fully developed condition. On the other hand, the injected polymer thread was subdivided considerably in the present experiments, and the polymer thread existed both in the buffer layer and the turbulent core region. We suppose, at the present stage, that the difference between the present experimental results and Berman and Sinha's data may be due to the difference in pipe diameters. It may be concluded that the interaction between polymer threads and turbulent eddies is quite different if the tube diameter is different.

\section{Concluding Remarks}

LDV measurements were carried out for both premixed and polymer injected drag-reducing tube flows. The increase in buffer layer thickness was of the same order in both systems. The energy spectra had the same tendency in that they increased in the lower frequency range and decreased in the higher frequency range from the Newtonian value. The difference in turbulent characteristics between premixed and polymer injected systems was observed in the distribution of turbulent macroscale. The enlargement of macroscale was more significant in the turbulent core region in the case of polymer injection experiments. The measurements of skewness and flatness factors showed that the low-speed fluid element caused by the ejection precess passed more clearly the measuring position near the outer boundary of the buffer layer in the case of polymer injection experiments.

We have observed that there existed some difference in turbulent characteristics between premixed and polymer injection systems. However, the drag reduction ratio was almost the same in both systems. It may be concluded that the polymer-turbulent eddy 
interaction is quite different between premixed and polymer injection systems. The detailed interaction between polymer thread and turbulent eddy was not clarified in this work. More accumulation of experimental evidence on this point is expected.

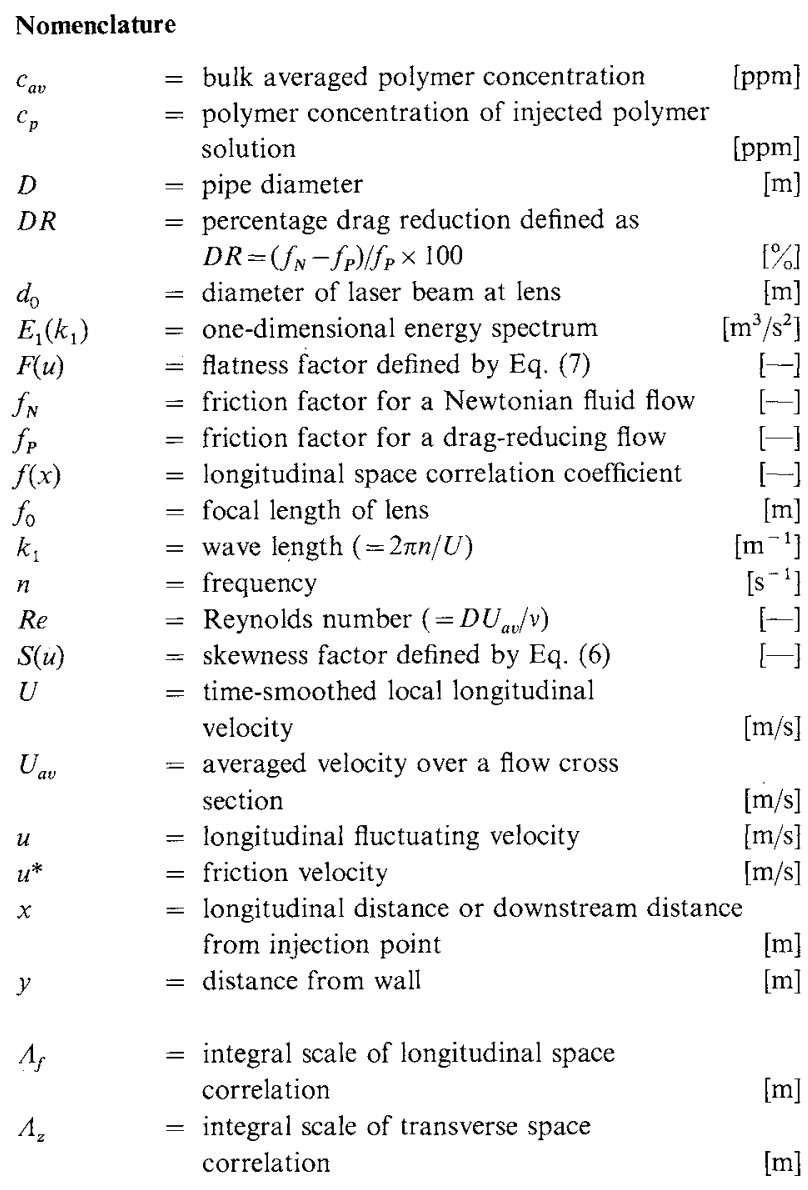

$\lambda \quad=$ wave length of laser beam $\quad[\mathrm{m}]$

$v \quad=$ kinematic viscosity $\left[\mathrm{m}^{2} / \mathrm{s}\right]$

$\sigma \quad=$ laser beam diameter at focal point $\quad[\mathrm{m}]$

$\langle$ Superscript〉

$\begin{aligned}+ & \text { nondimensionalized by wall parameters } \\ & \left(\text { i.e. } u^{*} \text { and } v \text { ) }\right.\end{aligned}$

\section{Literature Cited}

1) Achia, B. U. and D. W. Thompson: J. Fluid Mech., 81, 439 (1977).

2) Berman, N. S. and P. K. Sinha: Proc. 3rd Int. Conf. on Drag Reduction, ed. by R. H. J. Sellin and R. T. Moses, Univ. of Bristol, B-3 (1984).

3) Bewersdorff, H. W.: Proc. 3rd Int. Conf. on Drag Reduction, ed. by R. H. J. Sellin and R. T. Moses, Univ. of Bristol, B-4 (1984) or Rheol. Act., 21, 587 (1982).

4) El-Riedy, O. K. F. and B. Latto: Proc. 3rd Int. Conf. on Drag Reduction, ed. by R. H. J. Sellin and R. T. Moses, Univ. of Bristol, B-5 (1984).

5) Fortuna, G. and T. J. Hanratty: J. Fluid Mech., 53, 575 (1972).

6) George, W. and J. L. Lumley: J. Fluid Mech., 61, 321 (1973).

7) Kudva, A. K. and A. Sesonske: Int. J. Heat Mass Transfer, 15, 127 (1972).

8) Laufer, J.: NACA TN 2954 (1953).

9) McComb, W. D. and L. H. Rabie: AIChE J., 28, 547 (1982),

10) McComb, W. D. and L. H. Rabie: AIChE J., 28, 558 (1982).

11) Mizushina, T. and H. Usui: Phys. Fluids., 20, Suppl. 100 (1977).

12) Nakagawa, H. and I. Nezu: J. Fluid Mech., 80, 99 (1977).

13) Pennel, W. T., E. M. Sparrow and E. R. G. Eckert: Int J. Heat Mass Transfer, 15, 1067 (1972).

14) Reischman, M. M. and W. G. Tiederman: J. Fluid Mech., 70, 369 (1975).

15) Ueda, H. and J. O. Hinze: J. Fluid Mech. 67, 125 (1975).

16) Usui, H. and Y. Sano: AIChE J., 29, 611 (1983).

17) Virk, P. S.: AIChE J., 21, 625 (1975). 